\title{
Uma Estação de Medição Geomagnética de Acesso Ubíquo para Estudos de Geofísica
}

\author{
Fábio Franco de Oliveira', Rodolfo da Silva Villaça ${ }^{1}$, Celso Alberto Saibel Santos ${ }^{1}$ \\ Roberto Colistete Junior ${ }^{2}$, Jenesca Florencio Vicente Lima ${ }^{3}$, \\ ${ }^{1}$ Programa de Pós Graduação em Informática (PPGI) \\ Universidade Federal do Espírito Santo (UFES) \\ Vitória - ES - Brasil \\ ${ }^{2}$ Departamento de Química e Física \\ ${ }^{3}$ Departamento de Geologia \\ Universidade Federal do Espírito Santo (UFES) \\ Alegre - ES - Brasil
}

fabioti6@gmail.com, rodolfo.villaca@ufes.br, saibeldinf.ufes.br,
roberto.colistete@ufes.br, jenesca.lima@ufes.br

\begin{abstract}
Magnetometers are widely used in the field of geophysical survey for monitoring of planet's magnetic field and watching natural phenomena such as solar storms, earthquakes and tsunamis. Usually available on the market by a few manufacturers and with use of proprietary technologies, becomes a restricted equipment in emerging countries like Brazil - where it's cost is very high, beyond generally require extra equipment for monitoring measurements. This paper proposes the design and implementation of a geomagnetic station with low cost and open technologies which makes use of the Internet of Things to enable practical experiments in teaching of Physics and Geology in the Federal University of Espírito Santo.
\end{abstract}

Resumo. Magnetômetros têm sido amplamente usados no campo da pesquisa geofísica para o monitoramento do campo magnético do planeta e vigilância de fenômenos naturais como tempestades solares, terremotos e maremotos. Usualmente disponibilizado no mercado por poucos fabricantes e com uso de tecnologia proprietária, torna-se um equipamento de uso restrito em países emergentes como o Brasil - onde seu custo é bastante elevado, além de geralmente exigir equipamentos extras para o acompanhamento das medições. Este artigo apresenta o projeto e implementação de uma estação geomagnética de baixo custo, com tecnologias abertas e que faz uso da Internet das Coisas, viabilizando experiências práticas no ensino de Física e Geologia da Universidade Federal do Espírito Santo.

*Este trabalho tem recebido financiamento do projeto Horizon 2020 da União Europeia para pesquisa, desenvolvimento tecnológico e demonstração sob no. 688941 (FUTEBOL), assim como do Ministério Brasileiro da Ciência, Tecnologia e Inovação (MCTI) por meio da RNP e do CTIC. Alem disso, gostaríamos de agradecer o financiamento do CNPq sob no. 449369/2014-5, e da FAPES sob no. $524 / 2015$. 
V Congresso Brasileiro de Informática na Educação (CBIE 2016)

Anais dos Workshops do V Congresso Brasileiro de Informática na Educação (CBIE 2016)

\section{Introdução}

O planeta Terra, assim como os demais corpos celestiais, possui um núcleo composto de metais que podem chegar à temperaturas da ordem de $6000^{\circ} \mathrm{C}$, estando portanto sob a forma líquida. O fluído condutor, por estar em constante movimento de rotação e de convecção, termina por gerar e manter um campo magnético que se estende por milhares de quilômetros até o exterior do planeta, gerando assim o que chamamos de Magnetosfera.

A Figura 1 apresenta um diagrama mostrando como a Magnetosfera é formada a partir do núcleo do planeta, e reage, já na superfície da Terra, à exposição de ventos solares.

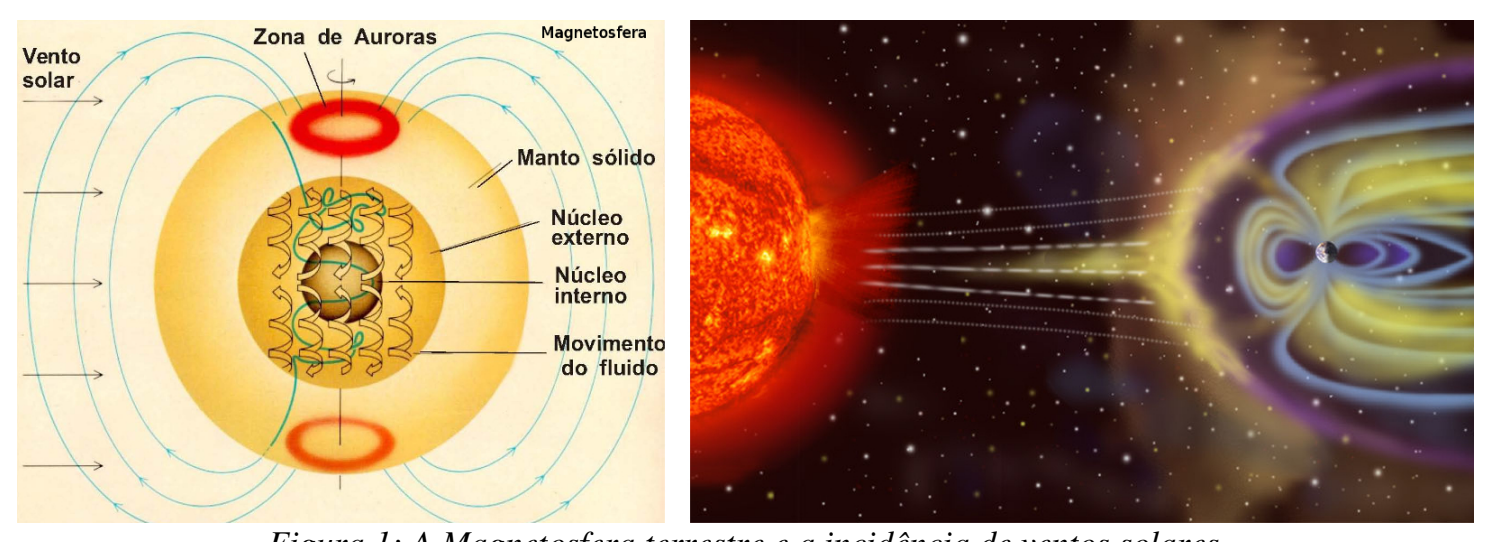

Figura 1: A Magnetosfera terrestre e a incidência de ventos solares (Fonte: Saint John's University / Geostationary Operational Environmental Satellite - NASA)

Situada acima da atmosfera terrestre, essa região funciona como um escudo que nos protege da alta carga de radiação emitida pelo Sol, chamada de Tempestade Solar ou simplesmente Ventos Solares [Space Weather Canada, 2016].

A exposição à radiação solar, além dos nossos já conhecidos problemas com raios ultravioleta, pode também interferir, de maneira sistemática, em satélites ou linhas de comunicação de dados, podendo chegar a extremos, como o ocorrido no ano de 1859 , que ficou conhecido como Evento Carrington. Naquele ano, segundo registros da [NASA, 2008], as descargas elétricas induzidas pelo magnetismo de uma forte tempestade solar provocaram a sobrecarga de linhas de telégrafo e o incêndio de inúmeros aparelhos conectados na Europa, causando choques elétricos em usuários e técnicos e gerando um caos na indústria das telecomunicações na época. A eventual ocorrência de um evento desses tornar a se repetir nos dias atuais, sem o devido acompanhamento quase dois séculos depois, com certeza traria consequências catastróficas.

Além do monitoramento da magnetosfera, geólogos e estudantes de geologia de escolas e universidades estudam e analisam diferentes tipos de formações rochosas e minerais magnéticos. A concentração destes materiais na crosta terrestre pode produzir distorções nas medições do campo magnético entre o valor mensurado e o valor esperado para aquela localidade [Oliveira, J. F, 1998] com base em modelos teóricos como o WMM e IGRF [National Oceanic and Atmospheric Administration, 2016]. 
Para o estudo e monitoramento da intensidade, direção e sentido de campos geomagnéticos, utiliza-se um equipamento chamado de Magnetômetro. Com este equipamento, é possível medir o campo magnético do planeta de forma a se acompanhar possíveis variações temporais causadas pela rotação da Terra (dia e noite), tempestades solares, pulsações internas na Terra, etc.

O objetivo deste trabalho é apresentar o projeto e a implementação de uma estação geomagnética com uso de componentes de baixo custo e tecnologias abertas, tornando a sua construção acessível para qualquer pesquisador. Além disso, o projeto prevê que os dados coletados sejam disponibilizados online por meio de uma arquitetura aderente ao modelo de Internet das Coisas (Internet of Things ou IoT). A expectativa é que com esse tipo de acesso facilitado aos dados, os estudantes se sintam mais estimulados (i) a pesquisarem sobre fenômenos físicos complexos, como a variação do campo geomagnético terrestre, e (ii) a interagirem com modelos experimentais baseados na coleta, processamento e análise de dados. Outro fato interessante é que o modelo de IoT adotado no projeto permite que professores, alunos e pesquisadores possam interagir de forma mais eficiente por meio do compartilhamento de dados coletados pelos mais diversos dispositivos através da Internet.

Para o desenvolvimento do trabalho será utilizado um microcomputador de placa única, um conversor analógico-digital de alta resolução, sensores magnéticos do tipo Fluxgate, conectividade Wireless, um relógio de tempo real e um sistema operacional de código fonte aberto.

$\mathrm{O}$ artigo visa apresentar o trabalho em andamento com a apresentação da arquitetura e os primeiros resultados do funcionamento do protótipo comparando os valores mensurados com os esperados para o campo magnético da localidade onde foram efetuados os testes. Na Seção 2, serão vistos quais os magnetômetros comerciais mais comuns para a mensuração de campos magnéticos. Na Seção 3, é feita uma discussão entre o modelo de arquitetura proprietária e aberta para a construção do equipamento. Na Seção 4, temos o descrição da arquitetura do magnetômetro com o detalhamento dos componentes que é feito na Seção 5. Finalmente, temos a apresentação dos resultados na Seção 6 e a conclusão do trabalho na Seção 7.

\section{Magnetômetros disponíveis atualmente}

Os magnetômetros, em geral, realizam a medição de magnetismo em Teslas (T). Construídos com diferentes tecnologias, de acordo com o grau de precisão requerido, $o$ tipo mais comum de magnetômetro é o construído com sensores do tipo Fluxgate, que se baseia no principio da indução mútua entre os sinais de uma bobina de excitação e uma bobina de detecção e o processamento do sinal de saída sobreposto entre eles [Waheed, O.T., 2011]. A Figura 2 mostra o diagrama básico de um sensor do tipo Fluxgate com um exemplo de um sensor deste tipo ao lado.
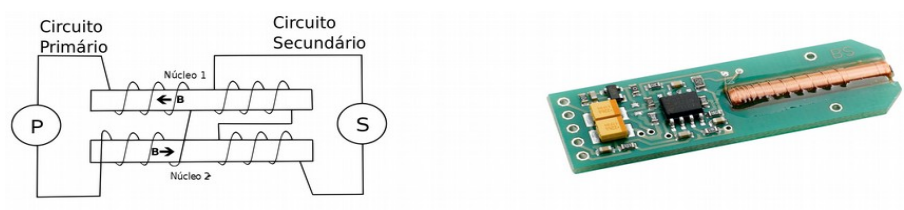

Figura 2: Magnetômetro do tipo Fluxgate

(Fonte: Wikimedia / Stefan Mayer Instruments) 
V Congresso Brasileiro de Informática na Educação (CBIE 2016)

Anais dos Workshops do V Congresso Brasileiro de Informática na Educação (CBIE 2016)

Existem equipamentos comerciais que empregam outros tipos de sensores, como os Magneto-resistivos e os de Efeito Hall (custo inferior com menor grau de precisão, sendo o primeiro usado em smartphones e tablets), Precessão de prótons (maior nível de precisão, com manutenção e uso mais complexos além de maior custo) e o SQUID (aquisição e construção restritos devido a complexidade e custo bastante elevado porém com o maior grau de precisão existente). Considerando que as leituras de campo geomagnético da Terra costumam ficar na faixa de 20 a $60 u \mathrm{~T}$ e no caso do Brasil na América Latina, uma média nominal de cerca de $23 u \mathrm{~T}$, consideramos o sensor Fluxgate o mais adequado para o propósito do projeto. Na Figura 3, temos magnetômetros que empregam diferentes tecnologias de sensores.

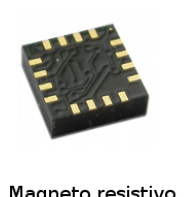

Magneto resistivo

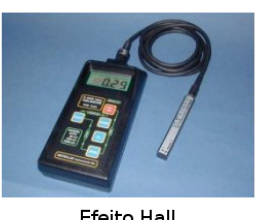

Efeito Hall

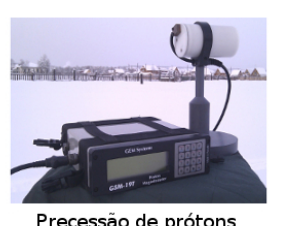

Precessão de prótons

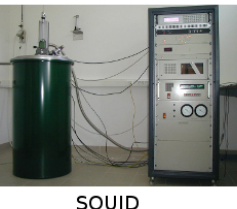

SQUID

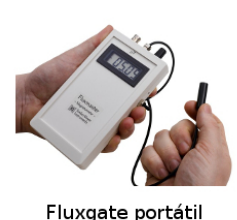

Fluxgate portátil

Figura 3: Tipos de magnetômetros

(Fonte: Wikimedia)

\section{Arquitetura Proprietária $\mathbf{X}$ Aberta}

A principal dificuldade para a ampliação do uso de magnetômetros comerciais nas mais diversas atividades, dentre as quais o ensino, é o seu custo. Atualmente, os preços deste tipo de equipamento se situam na faixa de $\mathrm{U} \$ 1250,00$ para modelos mais simples até U\$5900,00, para os mais avançados e precisos.

Considerando a resolução da ordem de 0,5 $n \mathrm{~T}$ dos equipamentos mais comuns disponíveis, fica claro que é possível de se construir uma ferramenta empregando componentes de baixo custo ou manufaturados artesanalmente que alcancem a precisão desejada. Finalmente, há a questão da arquitetura: os equipamentos comerciais disponíveis atualmente no mercado possuem arquitetura fechada, o que significa que os fabricantes não compartilham entre si o design interno de seus produtos tornando-os incompatíveis com outras soluções de hardware e de software. Uma arquitetura aberta, por outro lado, é concebida para facilitar o acréscimo, upgrade ou manutenção de componentes com facilidade. Possibilita ao usuário a customização de software com a adaptação e padronização de interfaces de usuário, integração com outros software tais como sistemas de monitoramento e controle [Júnior, F. F, 2005], sistemas de coleta e análise de dados com geração de históricos de informações e compartilhamento via Internet [Pritschow et al, 2001, Ferraz Jr. e Coelho, 2005]. Outro benefício do uso de sistemas abertos é a possibilidade de integração com outros sensores e sistemas de alerta de desastres naturais, conforme observado por [Okubo et al., 2011]. 
V Congresso Brasileiro de Informática na Educação (CBIE 2016)

Anais dos Workshops do V Congresso Brasileiro de Informática na Educação (CBIE 2016)

Tendo em vista os diversos benefícios, a proposta deste trabalho é verificar a viabilidade de construção de um magnetômetro de alta precisão (erro inferior a $5 n \mathrm{~T}$ ), usando equipamentos acessíveis e tecnologias abertas tais como um microcomputador de placa única, ADC e sistema operacional de código fonte aberto. Isto permite gerar versões customizadas da plataforma a um custo muito mais baixo do que os equipamentos comerciais, ampliando as possibilidades de uso desse tipo de equipamento. A plataforma terá acesso simplificado para a medição e registro dos dados a partir de um simples computador ou smartphone podendo beneficiar docentes e estudantes de cursos de graduação e pós graduação de áreas como a Física e Geologia.

\section{Arquitetura}

O magnetômetro a ser construído tem a arquitetura conforme a Figura 4:

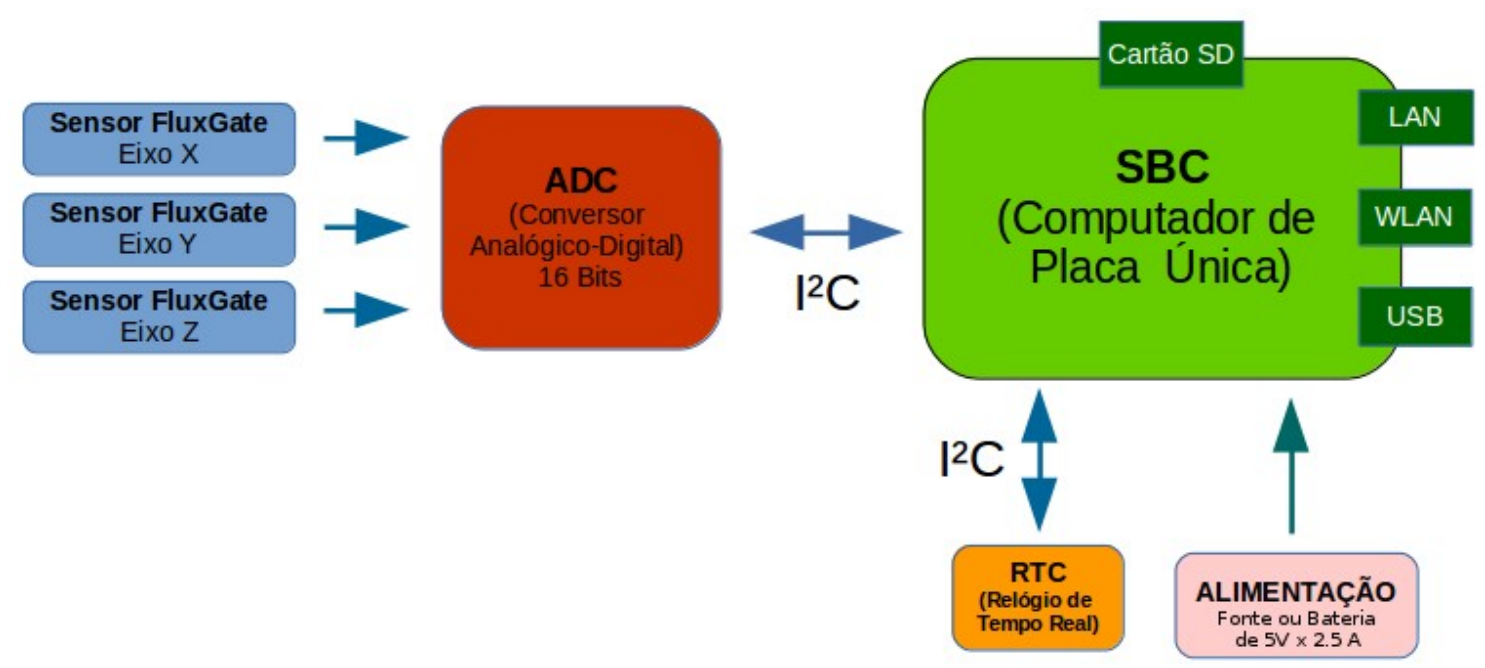

Figura 4: Diagrama da proposta do Magnetômetro

São usados 3 sensores magnéticos do tipo Fluxgate que possuem a função de monitorar o campo magnético terrestre em três eixos: Norte-Sul, Leste-Oeste e Vertical. Em suas saídas são geradas tensões (em Volts) proporcionais ao campo magnético mensurado. Os sensores são conectados a um conversor analógico digital, que é responsável pela quantização dos valores lidos a partir dos sensores. Por intermédio de um barramento $\mathrm{I}^{2} \mathrm{C}$ (Circuito Inter-Integrado) esses valores são entregues para um Computador de Placa Única (microcomputador) que, por sua vez, executa um sistema operacional embarcado de código-fonte aberto. O software desenvolvido monitora as leituras do ADC, efetuando a conversão dos valores para Teslas com cálculos de média, módulo e desvio padrão dos valores.

Os dados são registrados em um banco de dados SQL num cartão MicroSD e tornam-se disponíveis para acesso via rede local ou Internet. Para assegurar precisão no registro do tempo (data e hora) das medições, é usado também um módulo de Relógio de Tempo Real, regulado por cristal de quartzo, compensação por influência de temperatura e alimentação própria, fazendo com que o sistema mantenha seu horário sempre apurado mesmo em caso de interrupções na alimentação do equipamento. 
V Congresso Brasileiro de Informática na Educação (CBIE 2016)

Anais dos Workshops do V Congresso Brasileiro de Informática na Educação (CBIE 2016)

\section{Componentes}

O sensor Fluxgate responsável pelas leituras é o modelo FLC-100, fabricado pela [Stefan Mayer Instruments, 2016]. Possui as seguintes especificações:

- Saída em tensão proporcional ao campo magnético detectado

- Faixa de medição: $\pm 100 u \mathrm{~T}$

- Voltagem de operação: 5 Volts $\pm 5 \%$

- Consumo: $\sim 2 \mathrm{~mA}$

- $\quad$ Ruído: < 3 nTpp $(0,1$ a $10 \mathrm{~Hz})$

- Temperatura de operação: de -40 à $85^{\circ} \mathrm{C}$
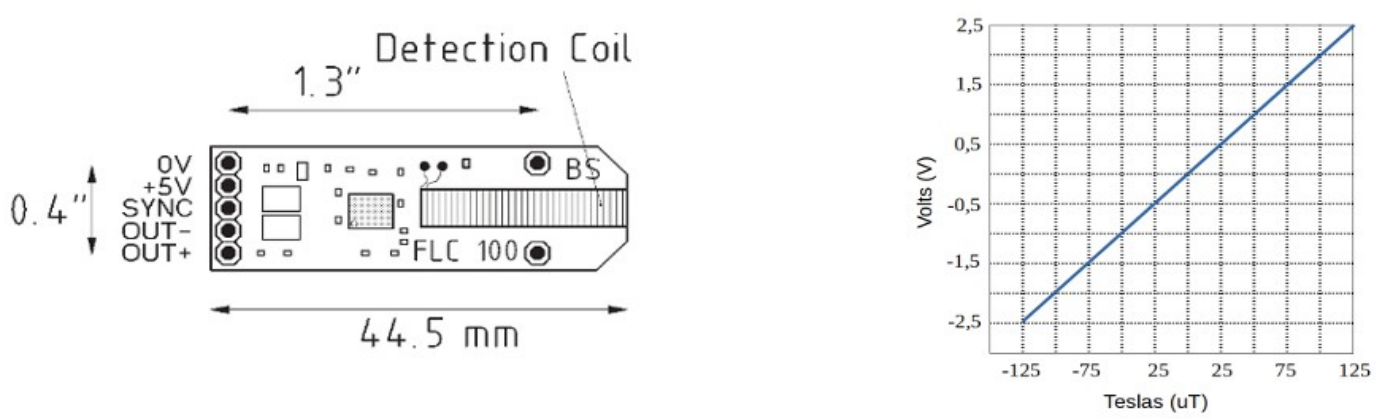

Figura 5: Especificações do sensor FLC-100. (Fonte: Stefan Mayer Instruments)

$\mathrm{Na}$ Figura 5, temos as especificações do sensor FLC-100 além de sua curva de transferência. Segundo os dados do fabricante [Stefan Mayer Instruments, 2016], a indução de um campo magnético de $50 u \mathrm{~T}$ gera na saída uma variação de voltagem de 1 Volt. Em testes em laboratório com uso de magnetos de neodímio e medições de distância, foi possível apurar que a resposta do sensor é linear em toda sua faixa de resposta.

Quanto ao conversor analógico-digital, o módulo a ser usado é o ADS1115 de 16 bits fabricado pela Texas Instruments que contém os seguintes recursos:

- Quatro canais de entrada com ganho ajustável

- Oscilador de Relógio

- Referência interna de voltagem

- Interface $\mathrm{I}^{2} \mathrm{C}$

- Taxa de amostragem entre 8 e 860 SPS

- Tensão de alimentação de 2,0 a 5,5 Volts

O Relógio de Tempo Real do projeto é o DS3231SN, que é dotado de um cristal oscilador com compensação térmica além de uma bateria de 3V do tipo CR2032 e uma interface $\mathrm{I}^{2} \mathrm{C}$. Sua precisão estimada é de $\pm 2 \mathrm{ppm}$ na faixa dos $0^{\circ} \mathrm{C}$ aos $40^{\circ} \mathrm{C}$. 
V Congresso Brasileiro de Informática na Educação (CBIE 2016)

Anais dos Workshops do V Congresso Brasileiro de Informática na Educação (CBIE 2016)

O Computador de Placa Única a ser utilizado na plataforma é o Raspberry PI 3 fabricado pela [Raspberry Pi Foundation, 2016]. O hardware é dotado das seguintes especificações conforme a Tabela 1:

Tabela 1. Especificações técnicas Raspberry Pi 3 Model B

(Fonte: Guia do usuário Raspberry Pi / RaspberryPi Foundation)

\begin{tabular}{ll}
\hline SoC (System on a Chip) & Broadcom BCM2837 \\
CPU & 1.2GHz 64-bit quad-core ARMv8 CPU \\
GPU & Dual Core VideoCore IV \\
Memória & $1024 \mathrm{Mb}$ SDRAM \\
Ethernet & Onboard 10/100 \\
Armazenamento & Micro SD \\
Consumo & $700 \mathrm{~mA}(3.5 \mathrm{~W})$ \\
Peso e Dimensões & 45 gramas $-8.6 \mathrm{~cm} \times 5.4 \mathrm{~cm} \times 1.7 \mathrm{~cm}$ \\
\hline
\end{tabular}

O sistema operacional utilizado no projeto é o Linux Raspbian Jessie, disponível para download também no site da [Raspberry Pi Foundation, 2016].

\section{Resultados}

Na etapa atual do projeto, verificou-se o grau de precisão do circuito ADC trabalhando com o sensor FLC-100. O objetivo desta etapa é verificar a precisão obtida em toda a faixa de leitura do sensor. O primeiro sensor a ser calibrado foi integrado ao projeto conforme a Figura 6.
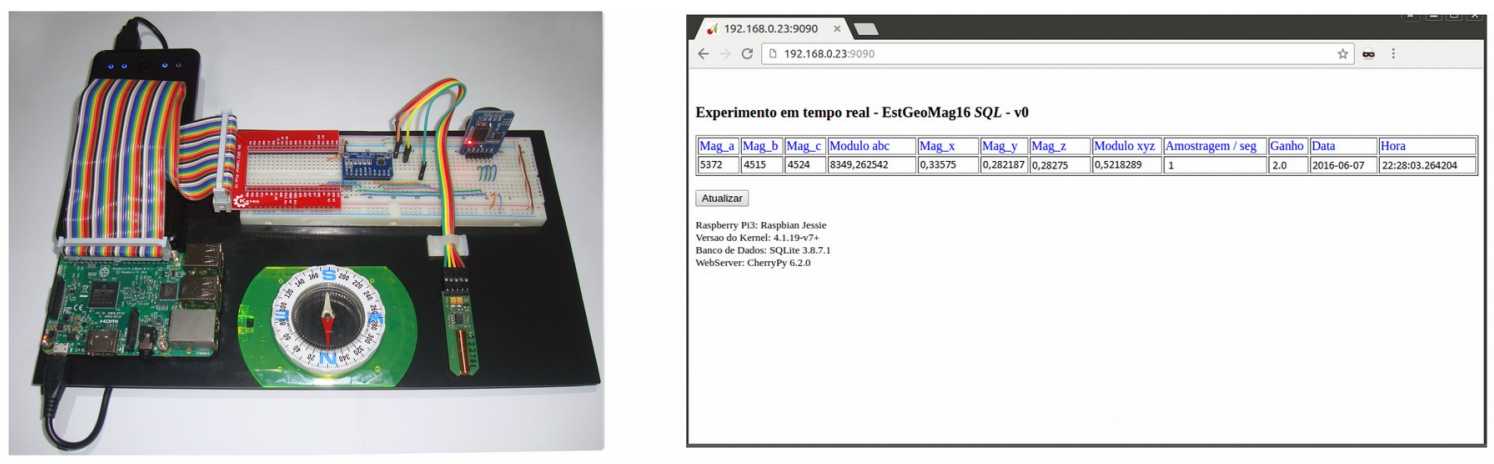

Figura 6: Protótipo da estação de medição / Exemplo de saída Web de dados lidos a partir do protótipo

Os dados coletados podem ser acessados através da rede por intermédio da interface Web utilizando o navegador de qualquer computador ou smartphone/tablet. Um exemplo de interface Web para acesso e apresentação dos dados é mostrado no lado direito da Figura 6. O acesso remoto à interface também foi testado com sucesso via Internet. 
V Congresso Brasileiro de Informática na Educação (CBIE 2016)

Anais dos Workshops do V Congresso Brasileiro de Informática na Educação (CBIE 2016)

Para o primeiro teste de funcionamento e precisão, foram feitas 1000 coletas pelo ADC numa taxa de 1 amostra por segundo. Considerando a Latitude e Longitude do local do experimento (Venda Nova do Imigrante - Espírito Santo), foram calculados no site da [National Oceanic and Atmospheric Administration, 2016] os valores estimados do campo magnético e suas componentes para o data de 07/06/2016 utilizando o modelo de referência WMM2015 [National Oceanic and Atmospheric Administration, 2016] conforme a Figura 7:

\begin{tabular}{|c|c|c|c|c|c|c|c|}
\hline \multicolumn{8}{|c|}{ Magnetic Field } \\
\hline Model Used: & WMM2015 & & & & & & \\
\hline Latitude: & $20^{\circ} 21^{\prime} 27.1^{\prime \prime} \mathrm{S}$ & & & & & & \\
\hline Date & $\begin{array}{l}\text { Declination } \\
(+E \mid-W)\end{array}$ & $\begin{array}{l}\text { Inclination } \\
(+D \mid-U)\end{array}$ & $\begin{array}{l}\text { Horizontal } \\
\text { Intensity }\end{array}$ & $\begin{array}{l}\text { North Comp } \\
\text { (+ N I-S) }\end{array}$ & $\begin{array}{l}\text { East Comp } \\
(+E \mid-W)\end{array}$ & $\begin{array}{l}\text { Vertical Comp } \\
\text { (+ D |-U) }\end{array}$ & Total Field \\
\hline
\end{tabular}

Figura 7: Tabela com dados do modelo disponibilizado NGDC

Apontando a estação minuciosamente em direção ao norte geomagnético da Terra com auxílio de uma bússola, o sinal obteve a amplitude mostrada na Figura 8 para as 1000 amostras/medições realizadas com o protótipo.

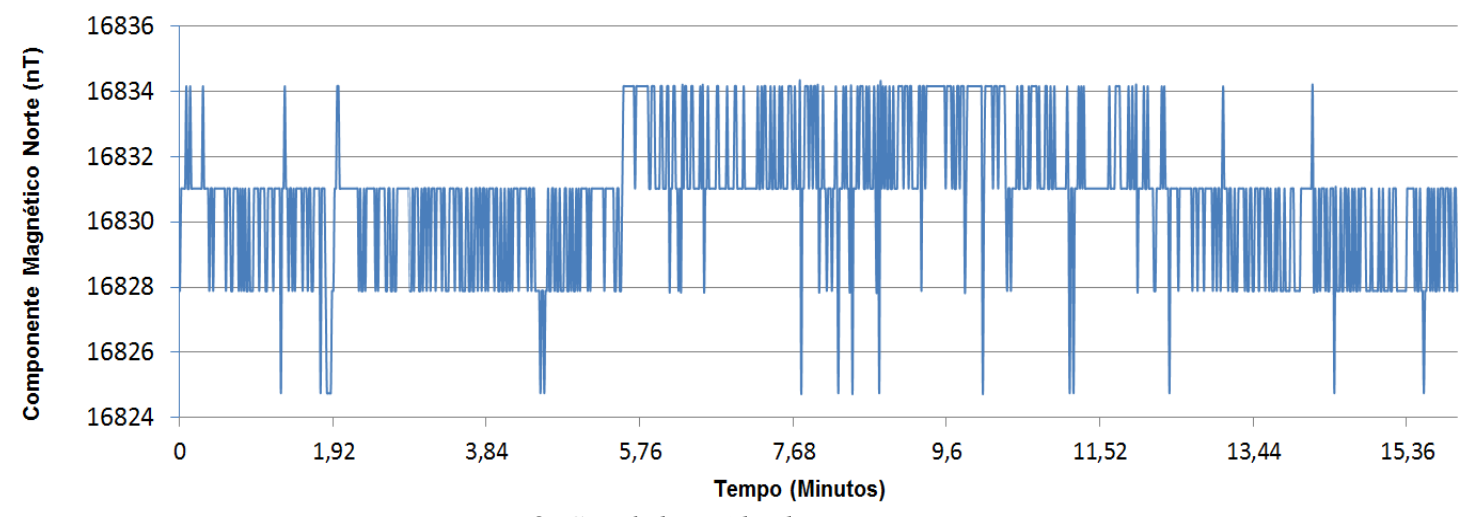

Figura 8: Sinal de saída da componente norte

Com variação máxima de $0,05 \%$ com relação ao valor esperado de $16831,1 n \mathrm{~T}$ (ver Figura 7, coluna North Comp) de magnitude para a componente norte, a plataforma atende à precisão pretendida do projeto ficando próximo ao valor do ruído de $3 n \mathrm{~T}$ da especificação [Stefan Mayer Instruments, 2016] do sensor FLC-100. 
V Congresso Brasileiro de Informática na Educação (CBIE 2016)

Anais dos Workshops do V Congresso Brasileiro de Informática na Educação (CBIE 2016)

\section{Conclusões}

Com a funcionalidade de acesso via web, a plataforma em estudos possui um grande potencial de aprendizagem para estudantes de cursos de Geologia e Física. O uso de tecnologias abertas desenvolvidas no contexto da Internet das Coisas possibilita o acesso à estações de geomonitoramento que podem estar dentro ou fora do Campus de uma universidade, podendo ser acessadas simultaneamente por toda uma turma, compartilhando portanto o mesmo equipamento e reduzindo custos.

Nos próximos passos o objetivo é monitorar simultaneamente os três eixos de vetores magnéticos via 3 magnetômetros Fluxgate FLC-100, obtendo assim o módulo resultante com maior precisão e menor desvio padrão com relação ao modelo WMM e IGRF. Também serão testados outros dois conversores ADC, um de 17 e outro de 24 bits, comparando com o ADC de 16 bits, visando ter uma leitura a mais precisa possível dos 3 magnetômetros Fluxgate FLC-100.

\section{Referências}

Waheed, O. T., Rehman, A. Design and Development of a Fluxgate

Magnetometer for Small Satellites in Low Earth Orbit. Journal of Space Technology, Vol 1, N. 1, p.78-2, 2011.

Júnior, F. F., de Oliveira, J. F. G., \& de Souza, A. F. Tecnologias de Supervisão e Monitoramento em Máquinas Ferramentas com CNC De Arquitetura Aberta. Congresso Brasileiro de Engenharia de Fabricação, 2005, Joinville. III - COBEF, 2005.

Pritschow, G. et al. Open Controller Architecture - Past, Present and Future. Annals of the CIRP, v. 50, n. 2, p. 1-7, 2001.

Ferraz JR, F.; COELHO, R. T. Data Acquisition and Monitoring in Machine Tools with CNC of Open Architecture using Internet. The International Journal of Advanced Manufacturing and Technology, v. 26, n. 1-2, p. 90, 2005.

Okubo, K. et al. Direct magnetic signals from earthquake rupturing: Iwate-Miyagi earthquake of M 7.2, Japan. Earth and Planetary Science Letters, v. 305, n. 1, p. 65-72, 2011.

Oliveira, J.F. Contribuições dos Métodos Geofísicos (Magnetometria, Gravimetria e Eletrorresistividade) à Geologia Costeira da Região Metropolitana de Fortaleza. Fortaleza, 1998. 111p. Dissertação de Mestrado - Centro de Ciências, UFC. 
V Congresso Brasileiro de Informática na Educação (CBIE 2016)

Anais dos Workshops do V Congresso Brasileiro de Informática na Educação (CBIE 2016)

Space Weather Canada. Geomagnetic Variations. Disponível em: <http://www.spaceweather.gc.ca/svren.php>. Acesso em 10 de Setembro de 2016.

National Aeronautics and Space Administration - NASA. Science News - A Super Solar Flare.

Disponível em: <http://science.nasa.gov/science-news/science-at-nasa/2008/06may_carringtonflare>.

Acesso em 07 de Setembro de 2016.

National Oceanic and Atmospheric Administration. Magnetic Field Estimated Values. Disponível em: $<$ http://www.ngdc.noaa.gov/geomag-web/\#igrfwmm>. Acesso em 7 de Junho de 2016.

National Oceanic and Atmospheric Administration. The World Magnetic Model. Disponível em: $<$ https://www.ngdc.noaa.gov/geomag/WMM/>. Acesso em 7 de Junho de 2016.

Stefan Mayer Instruments. Magnetic Field Sensor FLC 100. Disponível em: <http://www.stefanmayer.com/images/datasheets/Data-sheet FLC-100.pdf>. Acesso em 04 de Junho de 2016.

Raspberry PI Foundation. Raspberry PI Foundation Website. Disponível em: $<$ https://www.raspberrypi.org $>$. Acesso em 09 de Julho de 2016. 